\title{
Driven torsion pendulum for measuring the complex shear modulus in a steady shear flow
}

\author{
D. van den Ende, J. Mellema and C. Blom \\ Faculty of Applied Physics, University of Twente, The Netherlands
}

\begin{abstract}
To investigate the viscoelastic behavior of fluid dispersions under steady shear flow conditions, an apparatus for parallel superimposed oscillations has been constructed which consists of a rotating cup containing the liquid under investigation in which a torsional pendulum is immersed. By measuring the resonance frequency and bandwidth of the resonator in both liquid and in air, the frequency and steady-shear-rate-dependent complex shear modulus can be obtained. By exchange of the resonator lumps it is possible to use the instrument at four different frequencies: $85,284,740$, and $2440 \mathrm{~Hz}$ while the steady shear rate can be varied from 1 to $55 \mathrm{~s}^{-1}$. After treatment of the theoretical background, design, and measuring procedure, the calibration with a number of Newtonian liquids is described and the accuracy of the instrument is discussed.
\end{abstract}

Key words: Superimposed oscillatory shear; complex shear modulus; torsion pendulum; nearly viscometric flow

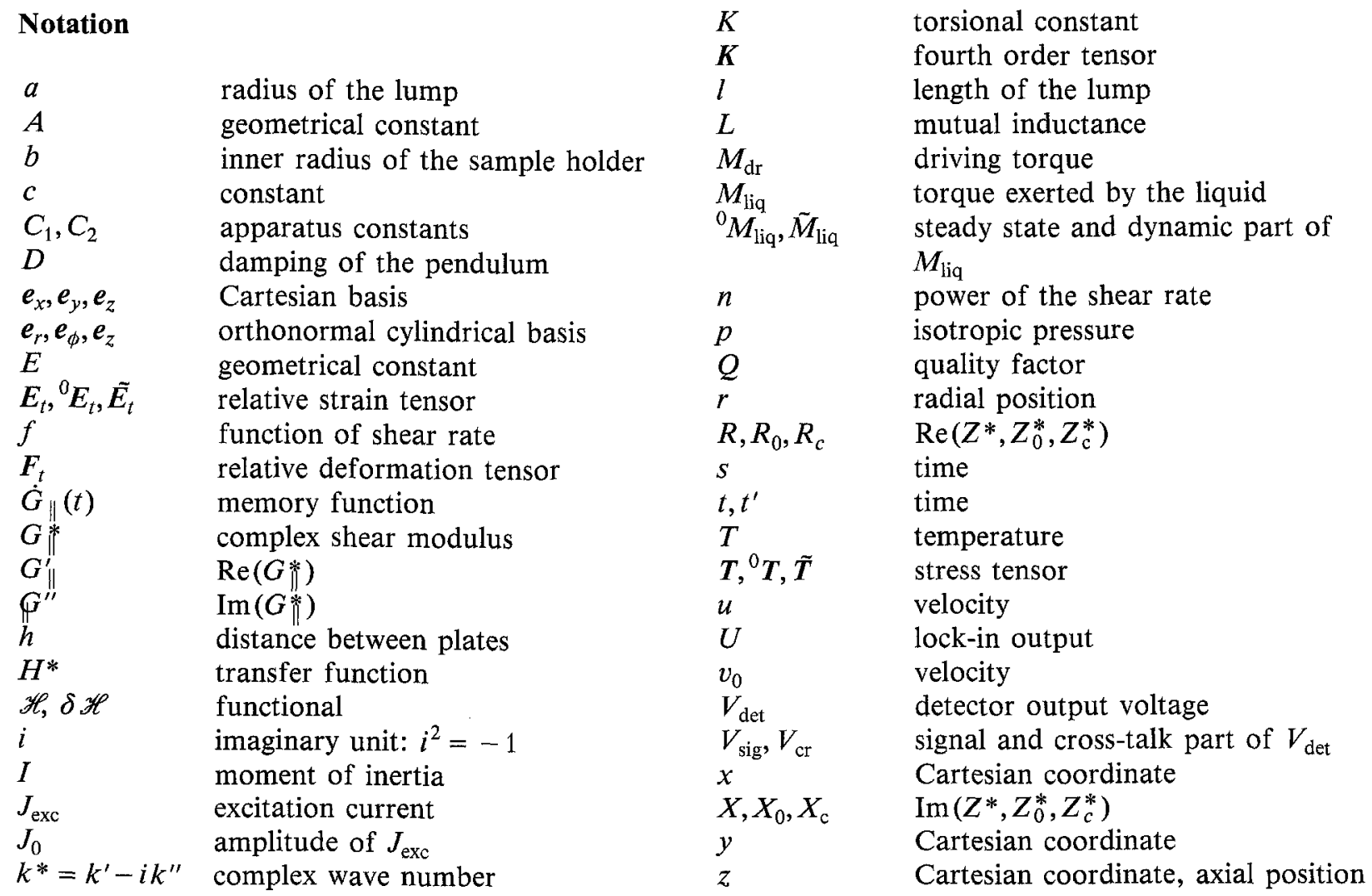




$\begin{array}{ll}Z^{*}, Z_{0}^{*}, Z_{c}^{*} & \text { impedance } \\ \alpha & \text { angle } \\ \dot{\gamma}_{0} & \text { steady shear rate } \\ \delta & \text { penetration depth } \\ \delta(t) & \text { Dirac delta function } \\ \varepsilon & \text { cylindrical angle } \\ \zeta & \text { phase angle } \\ \eta^{*}=\eta^{\prime}-i \eta^{\prime \prime} & \text { complex viscosity } \\ \vartheta & \text { cylinder angle } \\ \lambda & \text { wave length } \\ \xi & \text { relative displacement } \\ \xi_{0} & \text { amplitude of oscillation } \\ \pi & 3.14 \ldots \\ \varrho & \text { density } \\ \sigma & \text { dynamic shear stress } \\ \tau & \text { shear stress } \\ \tau_{0} & \text { steady-state shear stress } \\ \phi & \text { angular position } \\ \chi & \text { function of } \omega \\ \omega & \text { angular frequency } \\ \omega_{0}, \omega_{0}^{\text {vac }} & \text { resonance frequency } \\ \Delta \omega, \Delta \omega^{\text {vac }} & \text { bandwidth } \\ \Omega & \text { angular velocity }\end{array}$

\section{Introduction}

Linear viscoelastic measurements on non-Newtonian liquids (polymer melts and solutions, dispersions, emulsions, etc.) have proven to produce valuable data for the characterization of the micro structure in equilibrium of the liquid considered [7, 13, 16]. By superimposing small amplitude shear oscillations upon the steady shear flow, one is able to gain some insight into the steady shear flow microstructure. This technique was applied in the 1960 s on concentrated polymer solutions, mainly using commercially available instruments in the low-frequency regime $[3,4,8]$. Those instruments are not sensitive for fluid dispersions which exhibit a too small linear regime. These fluid dispersions exhibit, under steady flow, viscous and/or elastic effects which can be quite different from the linear rheological behavior at rest, due to changes in the microstructure. To investigate such behavior, an apparatus has been constructed with which one measures the complex dynamic shear modulus using superimposed oscillations upon a steady shear flow. The apparatus consists of a rotating cylindrical cup, containing the liquid under investigation, in which the cylindrical lump of a torsional pendulum is immersed coaxially. The measuring method is based upon the determination of the resonance frequency and the bandwidth of the resonator immersed in the liquid and in air, respectively. From the difference between the measurements in the two media the liquid impedance is obtained, and from this, the frequency and shear rate dependent shear moduli $G^{\prime}$ and $G^{\prime \prime}$ are calculated.

After a theoretical treatment in Sects. 2 and 3 of the relations between $G^{\prime}$ and $G^{\prime \prime}$ on the one hand and the resonance frequency and bandwidth on the other hand, the instrument and the measuring procedure are described in Sect. 4. In Sect. 5 the calibration of the apparatus with the help of some Newtonian liquids is presented and the accuracy obtained is discussed. The paper ends with a short discussion of the results in Sect. 6.

\section{Relation between the dynamic shear moduli and the measured liquid impedance}

In order to define the dynamic shear moduli in a steady state shear flow and to relate these moduli to the measured impedance, a general expression for the constitutive equation is taken as a starting point. According to the simple fluid theory of Truesdell and Noll [14] the stress in an incompressible fluid is a tensor valued functional of the history of the relative strain tensor:

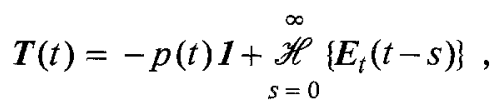

with $T(t)$ the stress tensor at time $t, p(t)$ the undetermined isotropic pressure at time $t, E_{t}(t-s)$ the relative strain tensor at time $t^{\prime}=t-s$, and $\mathscr{H}$ the tensor valued functional. The relative strain tensor can be expressed in the relative deformation gradient:

$$
\boldsymbol{E}_{t}(t-s)=\frac{1}{2}\left(\boldsymbol{F}_{t}^{T}(t-s) \boldsymbol{F}_{t}(t-s)-\boldsymbol{1}\right),
$$

with $\boldsymbol{F}_{t}$ the relative deformation gradient and $\boldsymbol{F}_{t}^{T}$ its transpose. In the case of a purely viscometric flow the stress tensor becomes a tensor valued function of the rate of strain. Superposition of a small amplitude oscillatory shear flow on this flow results in a nearly viscometric flow as defined by Pipkin and Owen [9]. For such flows the functional $\mathscr{H}$ can be expanded around ${ }^{0} E_{t}(t-s)$, the relative strain tensor in the pure viscometric case:

$$
\mathscr{H}\left\{E_{t}(t-s)\right\}=\mathscr{H}\left\{E_{t}(t-s)\right\}+\delta \mathscr{H}\left\{E_{i} ; \tilde{E}_{t}(t-s)\right\},
$$

with $\tilde{E}_{t}(t-s)=E_{t}(t-s)-{ }^{0} E_{t}(t-s)$ the oscillatory part of the relative strain tensor and $\delta \mathscr{H}$ the Fréchet 
differential of $\mathscr{H}$ at ${ }^{0} E_{t}[15]$. Higher orders in this expansion are omitted. Hence, the stress can be expressed as

$$
T(t)=-p I+{ }^{0} T+\tilde{T}(t),
$$

in which ${ }^{0} T$ the purely viscometric part of the stress and $\tilde{T}(t)=\delta \mathscr{H}\left\{{ }^{0} E_{t} ; \widetilde{E}_{t}(t-s)\right\}$ the nonviscometric part of the stress. This can be rewritten as [11]:

$$
\tilde{T}(t)=\int_{-\infty}^{\infty} K\left({ }^{0} E_{t}, s\right): \widetilde{E}_{t}(t-s) d s ;
$$

$\boldsymbol{K}$ is a fourth order tensor and the : indicates a double contraction. If ${ }^{0} E_{t}=0$ Eq. (5) reduces to the constitutive equation for a linear viscoelastic material. In the linear viscoelastic theory the tensor $K$ reduces to a scalar function due to the isotropy of the liquid under investigation. In the case of nearly viscometric flow, however, the isotropy of the liquid can be broken by the steady-state shear flow. It can be shown on symmetry-grounds that there exist 13 independent components [11].

The flow between parallel plates, as sketched in Fig. $1 \mathrm{a}$, is now regarded as a nearly viscometric flow. The steady-state flow field at time $t^{\prime}$ relative to time $t$ is characterized by

$$
\begin{aligned}
& x\left(t^{\prime}\right)=x(t)+\left(t^{\prime}-t\right) \dot{\gamma}_{0} y \\
& y\left(t^{\prime}\right)=y(t) \\
& z\left(t^{\prime}\right)=z(t),
\end{aligned}
$$

with respect to an orthonormal base $\left\{\boldsymbol{e}_{x}, \boldsymbol{e}_{y}, \boldsymbol{e}_{z}\right\}$. Here, $\dot{\gamma}_{0}=v_{0} / h$ is the steady-state shear rate. Using Eq. (2), ${ }^{0} E_{t}(t-s)$ is calculated:

$$
{ }^{0} \boldsymbol{E}_{t}(t-s)=-\frac{1}{2} s \dot{\gamma}_{0}\left(\boldsymbol{e}_{x} \boldsymbol{e}_{y}+\boldsymbol{e}_{y} \boldsymbol{e}_{x}\right)+\frac{1}{2} s^{2} \dot{\gamma}_{0}^{2} \boldsymbol{e}_{y} \boldsymbol{e}_{y} .
$$

Superimposing a small disturbance $\xi(t, y)$ (which is a function of $t$ and $y$ only) parallel to the steady-state shear flow results in

$$
\begin{aligned}
& x\left(t^{\prime}\right)=x(t)+\left(t^{\prime}-t\right) \dot{\gamma}_{0} y+\xi\left(t^{\prime}, y\right)-\xi(t, y) \\
& y\left(t^{\prime}\right)=y(t) \\
& z\left(t^{\prime}\right)=z(t) .
\end{aligned}
$$

Again, with Eq. (2) evaluation of $\tilde{E}_{t}(t-s)$ yields

$$
\begin{aligned}
\tilde{E}_{t}(t-s, y)= & \frac{1}{2}\left(\frac{\partial}{\partial y} \xi(t-s, y)\right. \\
& \left.-\frac{\partial}{\partial y} \xi(t, y)\right)\left(\boldsymbol{e}_{x} \boldsymbol{e}_{y}+\boldsymbol{e}_{y} \boldsymbol{e}_{x}\right) \\
& +\left[-s \dot{\gamma}_{0}\left(\frac{\partial}{\partial y} \xi(t-s, y)-\frac{\partial}{\partial y} \xi(t, y)\right)\right. \\
& \left.+\frac{1}{2}\left(\frac{\partial}{\partial y} \xi(t-s, y)-\frac{\partial}{\partial y} \xi(t, y)\right)^{2}\right] \boldsymbol{e}_{y} \boldsymbol{e}_{y}
\end{aligned}
$$
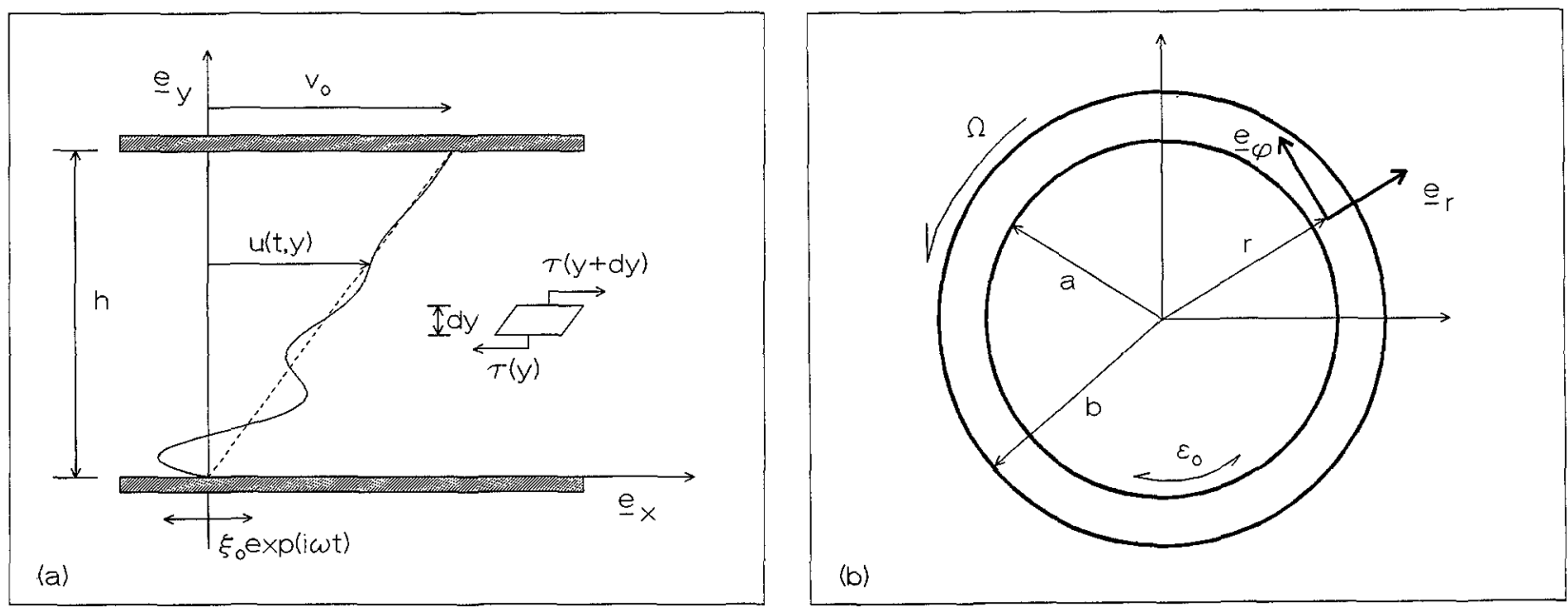

Fig. 1. Panel $a$ : The flow field between parallel plates; $h$ distance between plates, $v_{0}$ velocity of the upper plate, $\xi_{0}$ amplitude of oscillation of the lower plate, $\omega$ its angular frequency, $u(t, y)$ the velocity at height $y$ and time $t, \tau$ the shear stress. Panel $b$ : The flow field between concentric cylinders; $a$ is the radius of the inner cylinder, $b$ the radius of the outer cylinder, $\Omega$ the angular velocity of the outer cylinder, $\varepsilon_{0}$ the angular amplitude of oscillation of the inner cylinder, $\omega$ its angular frequency, $r$ the radial position in the liquid and $e_{r}$, $e_{\phi}$ unit vectors of the orthonormal basis 
In the geometry considered, only the $\langle x y\rangle$ component of the stress tensor is involved. Evaluating this component, one obtains from Eq. 5):

$$
\begin{aligned}
\tilde{T}_{x y}(t)= & \int_{-\infty}^{\infty}\left[K_{x y x y}\left(\dot{\gamma}_{0}, s\right)-\dot{\gamma}_{0} s K_{x y y y}\left(\dot{\gamma}_{0}, s\right)\right] \\
& \times\left[\frac{\partial}{\partial y} \xi(t-s, y)-\frac{\partial}{\partial y} \xi(t, y)\right] d s .
\end{aligned}
$$

In the evaluation the symmetry-relation: $K_{x y x y}=$ $k_{x y y x}$ has been used and the quadratic term in $\left[\frac{\partial}{\partial y} \xi(t-s, y)-\frac{\partial}{\partial y} \xi(t, y)\right]$ (which is small with respect to its linear term) is neglected. Defining

$$
\begin{aligned}
\dot{G}_{\|}\left(\dot{\gamma}_{0}, s\right)= & K_{x y x y}\left(\dot{\gamma}_{0}, s\right)-\dot{\gamma}_{0} s K_{x y y y}\left(\dot{\gamma}_{0}, s\right) \\
& -\int_{-\infty}^{\infty}\left[K_{x y x y}\left(\dot{\gamma}_{0}, s^{\prime}\right)\right. \\
& \left.-\dot{\gamma}_{0} s^{\prime} K_{x y y y}\left(\dot{\gamma}_{0}, s^{\prime}\right)\right] d s^{\prime} \delta(s),
\end{aligned}
$$

with $\delta(t)$ the Dirac delta function, Eq. (10) reduces to:

$$
\tilde{T}_{x y}(t, y)=\int_{-\infty}^{\infty} \dot{G}_{\|}\left(\dot{\gamma}_{0}, s\right) \cdot \frac{\partial}{\partial y} \xi(t-s, y) d s
$$

From the last expression, it is obvious that the nonviscometric oscillatory parts of the stress and strain can be handled as in the linear viscoelastic theory. Thus, the complex shear modulus $G^{*}\left(\dot{\gamma}_{0}, \omega\right)$ is defined as the Fourier Transform of $\dot{G}_{\|}\left(\dot{\gamma}_{0}, t\right)$.

The next step is the calculation of the shear stress exerted by the liquid on the oscillating plate. Considering a fluid element, as indicated in Fig. $1 \mathrm{a}$, combining Eqs. (4) and (12) with the equation of motion: $\varrho \frac{\partial u}{\partial t}=\frac{\partial \tau}{\partial y}$, in which $u(t, y)$ is the velocity (in $x$-direction) and $\tau=T_{x y}$, yields

$$
\begin{aligned}
& \varrho\left(\frac{\partial}{\partial t}\right)^{2} \xi(t, y) \\
& \quad=\left(\frac{\partial}{\partial y}\right)^{2} \int_{-\infty}^{\infty} \dot{G}_{\|}\left(\dot{\gamma}_{0}, s\right) \xi(t-s, y) d s .
\end{aligned}
$$

The boundary conditions for this geometry are given by

$$
\begin{aligned}
& \xi(t, y=0)=\xi_{0} e^{i \omega t} \\
& u(t, y=h)=v_{0},
\end{aligned}
$$

where $i^{2}=-1$ and $\omega$ is the angular frequency of the oscillating plate. In order to obtain the solution for this problem, one can start with a trial function $\xi(t, y)=\bar{\xi}(y) e^{i \omega t}$. The partial differential equation then reduces to the well known harmonic equation:

$$
\left(\frac{\partial}{\partial y}\right)^{2} \hat{\xi}(y)+\left(k^{*}\right)^{2} \cdot \hat{\xi}(y)=0 .
$$

Writing down this expression, one introduces the complex number $k^{*}=k^{\prime}-i k^{\prime \prime}$ with $k^{\prime \prime} \geq 0$, defined by:

$$
\left(k^{*}\right)^{2}=\varrho \omega^{2} / G^{*}\left(\dot{\gamma}_{0}, \omega\right) .
$$

The formal solution is given by

$$
\xi(t, y)=\xi_{0} e^{i \omega t} \frac{\sin \left[k^{*}(h-y)\right]}{\sin \left(k^{*} h\right)},
$$

representing a shear wave induced by the oscillating plate with wavelength $\lambda=2 \pi / k^{\prime}$ and penetration depth $\delta=1 / k^{\prime \prime}$. Now, the oscillatory part of the shear stress $\sigma=\tilde{T}_{x y}$ is obtained from Eq. (12). At $y=0$, this is the oscillatory part of the stress on the oscillating plate:

$$
\sigma(t, y=0)=-\left(\varrho \omega^{2} / k^{*}\right) \cot \left(k^{*} h\right) \xi_{0} e^{i \omega t} .
$$

The total stress on the plate is

$$
\tau(t, y=0)={ }^{0} T_{x y}\left(\dot{\gamma}_{0}\right)+\sigma(t, y=0) .
$$

Similarly, as in the analysis of the linear viscoelastic case [5], the characteristic impedance of the liquid on the plate is defined by $Z_{0}^{*}=-\sigma(t, y=0) / \xi(t, y=0)$. With Eqs. (17) and (18) in mind, one obtains for this impedance

$$
Z_{0}^{*}=\frac{\varrho \omega}{i k^{*}} \cot \left(k^{*} h\right)
$$

In the case of surface loading the induced shear wave is damped out before it reaches the other plate at $y=h: k^{\prime \prime} h \gg 1$, thus, the expression for the impedance reduces to

$$
Z_{0}^{*}=\varrho \omega / k^{*} .
$$

In the apparatus considered in this paper the loading on the lump of the surrounding liquid is composed of two parts, one due to the planar shear waves 
from the top and bottom of the lump, the second due to the cylindrical shear waves from the side. Thus, an expression for the cylindrical impedance is needed, too. Therefore, the general expressions in Eqs. (1) -(5) are examined for a cylindrical geometry in which the outer cylinder rotates with a constant angular velocity and the inner one describes a small amplitude oscillatory angular motion, as sketched in Fig. $1 \mathrm{~b}$. The flow field at time $t^{\prime}$ relative to time $t$ is, in this case, given by

$$
\begin{aligned}
& \phi\left(t^{\prime}\right)=\phi(t)+\left(t^{\prime}-t\right) f(r)+\varepsilon\left(t^{\prime}, r\right)-\varepsilon(t, r) \\
& r\left(t^{\prime}\right)=r(t) \\
& z\left(t^{\prime}\right)=z(t),
\end{aligned}
$$

with respect to the orthonormal base $\left\{\boldsymbol{e}_{\phi}, \boldsymbol{e}_{r}, \boldsymbol{e}_{z}\right\}$ for cylindrical coordinates. Here, $f(r)$ is the viscometric part of the angular velocity of the fluid at a distance $r$ from the axis. Evaluation of the viscometric and the oscillatory parts of the relative strain results in

$$
{ }^{0} \boldsymbol{E}_{t}(t-s)=-\frac{1}{2} s \dot{\gamma}_{0}\left(\boldsymbol{e}_{\phi} \boldsymbol{e}_{r}+\boldsymbol{e}_{r} \boldsymbol{e}_{\phi}\right)+\frac{1}{2} s^{2} \dot{\gamma}_{0}^{2} \boldsymbol{e}_{r} \boldsymbol{e}_{r},
$$

where $\boldsymbol{e}_{\phi}$ and $\boldsymbol{e}_{r}$ are considered at time $t$, and

$$
\begin{aligned}
\tilde{E}_{t}(t-s)= & \frac{1}{2} r\left(\frac{\partial}{\partial r} \varepsilon(t-s)-\frac{\partial}{\partial r} \varepsilon(t)\right)\left(\boldsymbol{e}_{\phi} \boldsymbol{e}_{r}+\boldsymbol{e}_{r} \boldsymbol{e}_{\phi}\right) \\
& -s \dot{\gamma}_{0} r\left(\frac{\partial}{\partial r} \varepsilon(t-s)-\frac{\partial}{\partial r} \varepsilon(t)\right) \boldsymbol{e}_{r} \boldsymbol{e}_{r}
\end{aligned}
$$

The steady-state shear rate $\dot{\gamma}_{0}$ is related to $f(r)$ according to $\dot{\gamma}_{0}(r)=r \partial f / \partial r$. The $\langle\phi r\rangle$ component of the stress tensor $\tilde{T}$ is obtained in the same way as $\tilde{T}_{x y}$ in Eq. (12), using Eq. (5):

$$
\widetilde{T}_{\phi r}(t, r)=\int_{-\infty}^{\infty} \dot{G}_{\|}\left(\dot{\gamma}_{0}(r), s\right) r \frac{\partial}{\partial r} \varepsilon(t-s, r) d s
$$

and the equation of motion: $\varrho \frac{\partial u}{\partial t}=\left(1 / r^{2}\right) \frac{\partial}{\partial r}\left[r^{2} \tau(r)\right]$, with $\tau=T_{\phi r}$, and $u$ the velocity in $\phi$-direction is transformed to

$$
\begin{aligned}
& \varrho\left(\frac{\partial}{\partial t}\right)^{2} \varepsilon(t, r) \\
& \quad=\left(\frac{1}{r}\right)^{3} \frac{\partial}{\partial r} \int_{-\infty}^{\infty} r^{3} \dot{G}_{\|}\left(\dot{\gamma}_{0}(r), s\right) \frac{\partial}{\partial r} \varepsilon(t-s, r) d s,
\end{aligned}
$$

with boundary conditions

$$
\begin{aligned}
& \varepsilon(t, r=a)=\varepsilon_{0} e^{i \omega t} \\
& \varepsilon(t, r=b)=0,
\end{aligned}
$$

where $a$ is the radius of the lump and $b=a+h$ being the radius of the outer cylinder.

Note that for $\dot{\gamma}_{0} \neq 0, \dot{G}_{\|}\left(\dot{\gamma}_{0}(r), t\right)$ implicitly depends on $r$. Therefore, the situation for $\dot{\gamma}_{0}=0$, where $\dot{G}$ and $k^{*}$ are independent of $r$, will be considered first. Using a trial function $\varepsilon(t, r)=\hat{\varepsilon}(r) e^{i \omega t}$ and defining $\hat{\xi}(r)=r \hat{\varepsilon}(r)$ evaluation of Eq. (26) gives the Bessel equation of order 1 :

$$
\left(\frac{\partial}{\partial r}\right)^{2} \hat{\xi}(r)+\frac{1}{r} \frac{\partial}{\partial r} \hat{\xi}(r)+\left\{\left(k^{*}\right)^{2}-\left(\frac{1}{r}\right)^{2}\right\} \hat{\xi}(r)=0 .
$$

A formal solution of this equation which fulfills the boundary conditions is given by:

$$
\hat{\xi}(r)=a \varepsilon_{0} \frac{H_{1}^{(2)}\left(k^{*} r\right) H_{1}^{(1)}\left(k^{*} b\right)-H_{1}^{(2)}\left(k^{*} b\right) H_{1}^{(1)}\left(k^{*} r\right)}{H_{1}^{(2)}\left(k^{*} a\right) H_{1}^{(1)}\left(k^{*} b\right)-H_{1}^{(2)}\left(k^{*} b\right) H_{1}^{(1)}\left(k^{*} a\right)},
$$

where $H_{n}^{(1)}(x)$ and $H_{n}^{(2)}(x)$ are Hankel functions of the first and second kind of order $n$. The oscillatory part of the shear stress $\sigma=\widetilde{T}_{\phi r}$ on the lump $r=a$ can be obtained using Eq. (25):

$$
\sigma(t, a)=G_{\|}^{*} \varepsilon_{0}\left\{k^{*} a \frac{H_{1}^{(2) \prime}\left(k^{*} a\right) H_{1}^{(1)}\left(k^{*} b\right)-H_{1}^{(2)}\left(k^{*} b\right) H_{1}^{(1) \prime}\left(k^{*} a\right)}{H_{1}^{(2)}\left(k^{*} a\right) H_{1}^{(1)}\left(k^{*} b\right)-H_{1}^{(2)}\left(k^{*} b\right) H_{1}^{(1)}\left(k^{*} a\right)}-1\right\} e^{i \omega t},
$$

where $H_{1}^{(1) \prime}(x)$ and $H_{1}^{(2) \prime}(x)$ are the derivatives of $H_{1}^{(1)}(x)$ and $H_{1}^{(2)}(x)$, respectively. Applying the rules for differentiation of Hankel functions as given by Abramowitz and Stegun [1], one obtains for the characteristic cylindrical impedance $Z_{c}^{*}=-\sigma(t, r=a) /(a \cdot \dot{\varepsilon}(t, r=a))$ : 


$$
Z_{c}^{*}=\frac{G_{1}^{*}}{i \omega a}\left\{2-k^{*} a \frac{H_{0}^{(2)}\left(k^{*} a\right) H_{1}^{(1)}\left(k^{*} b\right)-H_{1}^{(2)}\left(k^{*} b\right) H_{0}^{(1)}\left(k^{*} a\right)}{H_{1}^{(2)}\left(k^{*} a\right) H_{1}^{(1)}\left(k^{*} b\right)-H_{1}^{(2)}\left(k^{*} b\right) H_{1}^{(1)}\left(k^{*} a\right)}\right\} .
$$

The last expression is difficult to evaluate numerically because of the complex arguments of the Hankel functions involved. If the radius of the lump is not to small, $\left|k^{*} a\right| \gg 1$, an expansion of the Hankel functions for large arguments [1] is appropriate and Eq. (31) reduces to:

$$
\begin{aligned}
Z_{c}^{*}= & \frac{\varrho \omega}{i k^{*}}\left\{\cot \left[k^{*}(b-a)\right]\right. \\
& +\frac{1}{8 k^{*} a}\left\{15+3 \cot ^{2}\left[k^{*}(b-a)\right]\right\} \\
& \left.-\frac{3}{8 k^{*} b}\left\{1+\cot ^{2}\left[k^{*}(b-a)\right]\right\}\right\} .
\end{aligned}
$$

In the case of nearly surface loading, $k^{\prime \prime}(b-a) \gg 1$, the impedance is approximated $\left[\cot (z) \simeq i\left(1+2 e^{-2 i z}\right)\right.$ and $\left.\cot ^{2}(z) \simeq-\left(1+4 e^{-2 i z}\right)\right]$ by

$$
\begin{aligned}
Z_{c}^{*} & \simeq\left(\frac{\varrho \omega}{k^{*}}\right)\left\{1-\frac{3 i}{2 \varrho \omega a}\left(\frac{\varrho \omega}{k^{*}}\right)\right. \\
& \left.+2 e^{-2 i k^{*}(b-a)}\left[1+\frac{3 i}{4 \varrho \omega}\left(\frac{b-a}{a b}\right)\left(\frac{\varrho \omega}{k^{*}}\right)\right]\right\} .
\end{aligned}
$$

This is equivalent to the expression given by Schrag and Johnson [12], who neglected the second boundary and so omitted the term with $e^{-2 i k^{*}(b-a)}$.

In order to obtain a first-order correction due to the $r$-dependence of $\dot{G}_{\|}\left(\dot{\gamma}_{0}(r), t\right)$ the curvature of the geometry is ignored by considering $r^{3}$ in Eq. (26) as a constant, which is equivalent with the case of parallel plates, but with a $y$-dependent $\dot{G}_{\|}$. Then, Eq. (26) reduces to:

$$
\begin{aligned}
& \varrho\left(\frac{\partial}{\partial t}\right)^{2} \xi(t, r) \\
& \quad=\left(\frac{\partial}{\partial r}\right) \int_{-\infty}^{\infty} \dot{G}_{\|}\left(\dot{\gamma}_{0}(r), s\right)\left(\frac{\partial}{\partial r}\right) \xi(t-s, r) d s,
\end{aligned}
$$

where $\xi(t, r)=r \varepsilon(t, r)$. With the factorization $\xi(t, r)=\hat{\xi}(r) e^{i \omega t}$, this is equivalent to

$$
-\omega^{2} \varrho \bar{\xi}(r)=\frac{\partial}{\partial r}\left\{G^{*}\left(\dot{\gamma}_{0}(r), \omega\right) \frac{\partial}{\partial r} \hat{\xi}(r)\right\} .
$$

Defining $H(r)$ as a primitive of $\hat{\xi}(r)$, Eq. (35) can be written as

$$
\left(\frac{\partial}{\partial r}\right)^{2} H(r)+\left[k^{*}(r)\right]^{2} H(r)=0
$$

where $k^{*}(r)$ has been defined in Eq. (16) and is a function of $r$. Using the WKB approximation [6], one states: $H(r)=A e^{i \Phi(r)}$. Then the following approximate solutions for $H(r)$ and $\hat{\xi}(r)$ are obtained:

$$
\begin{aligned}
& H(r) \simeq A\left[k^{*}(r)\right]^{-1 / 2} \exp \left\{-i \int_{a}^{r} k^{*}\left(r^{\prime}\right) d r^{\prime}\right\} \\
& \xi(r) \simeq \xi_{0}\left(\frac{k^{*}(r)}{k^{*}(a)}\right)^{1 / 2} \\
& \times \frac{1-i / 2\left[k^{*}(r)\right]^{-2} \frac{\partial k^{*}}{\partial r}}{1-i / 2\left[k^{*}(a)\right]^{-2}\left(\frac{\partial k^{*}}{\partial r}\right)_{r=a}} \exp \left\{-i \int_{a}^{r} k^{*}\left(r^{\prime}\right) d r^{\prime}\right\}
\end{aligned}
$$

In the last expressions surface loading is assumed. These solutions are valid as long as

$$
\left|\frac{1}{2}\left[k^{*}(r)\right]^{-2}\left(\partial k^{*} / \partial r\right)\right|^{2} \ll 1,
$$

which means that the variation of both the wavelength and the penetration depth with $r$ has to be sufficiently small. With Eq. (38) the characteristic impedance $Z_{c}^{*}$ can be calculated as before:

$$
Z_{c}^{*} \simeq \frac{\varrho \omega}{k^{*}(a)}\left\{1-i / 2\left[k^{*}(a)\right]^{-2}\left(\partial k^{*} / \partial r\right)_{r=a}\right\} .
$$

Considering now the first-order corrections due to the 
curvature and due to the $r$-dependence of $\dot{\gamma}_{0}$ [Eqs. (33) and (40)], one finally obtains

$$
\begin{aligned}
& Z_{c}^{*}=\frac{\varrho \omega}{k^{*}(a)} \\
& \times\left\{1-\frac{3 i}{2 \varrho \omega a}\left(\frac{\varrho \omega}{k^{*}(a)}\right)\left[1+\frac{a}{3 k^{*}(a)}\left(\frac{\partial k^{*}}{\partial r}\right)_{r=a}\right]\right\} .
\end{aligned}
$$

Then the total impedance on the lump $Z^{*}=$ $-\tilde{M}_{\text {liq }} / \dot{\varepsilon}_{c}$, where $\tilde{M}_{\text {liq }}$ is the dynamic part of the torque exerted on the lump is given by

$$
Z^{*}=2 \pi a^{3} l Z_{c}^{*}+\frac{\pi a^{4}}{\cos \alpha} Z_{0}^{*},
$$

where $Z_{0}^{*}=\varrho \omega / k^{*}(a)$, while $l$ is the length of the cylindrical part of the lump and $\alpha$ a small angle the top and bottom surfaces make with a plane perpendicular to the pendulum axis (see Fig. 2). With Eq. (21) and the definition of $k^{*}$ [Eq. (16)], one finally obtains the expression relating $G_{\|}^{*}$ and $Z^{*}$ :

$$
\begin{aligned}
& G_{\|}^{*}\left(\dot{\gamma}_{0}(a), \omega\right) \simeq \frac{1}{\varrho}\left(\frac{A}{2 I} Z^{*}\right)^{2} \\
& \quad \times\left(1+2 i \frac{E}{\omega \varrho} \frac{A}{2 I} Z^{*}\left[1+\frac{a}{3 k^{*}(a)}\left(\frac{\partial k^{*}}{\partial r}\right)_{r=a}\right]\right),
\end{aligned}
$$

where $A / 2 I=\left(2 \pi a^{3} l+\pi a^{4} / \cos \alpha\right)^{-1}$ and $E=3 l /$ $\left(2 a l+a^{2} / \cos \alpha\right)$ are geometrical constants of the pendulum and $I$ is the moment of inertia of the pendulum mass. In Eq. (43) $G_{\|}^{*}$ is implicitly given due to the last term in the square brackets. Therefore, $G_{\|}$ has to be calculated in an iterative way, starting with $\partial k^{*} / \partial r=0$. In most cases $a\left(\partial k^{*} / \partial r\right)_{r=a} /\left[3 k^{*}(a)\right]$ $<1$, and the series of solutions converges very rapidly. The complex viscosity $\eta_{\|}^{*}$ can be calculated with the help of: $G_{\|}^{*}\left(\dot{\gamma}_{0}, \omega\right)=i \omega \eta_{\|}^{*}\left(\dot{\gamma}_{0}, \omega\right)$.

\section{From resonance frequency and bandwidth to the liquid impedance}

As one observes from the diagrams in Fig. 2, the lump, which contains a permanent magnet, is driven by two excitation coils positioned just outside the

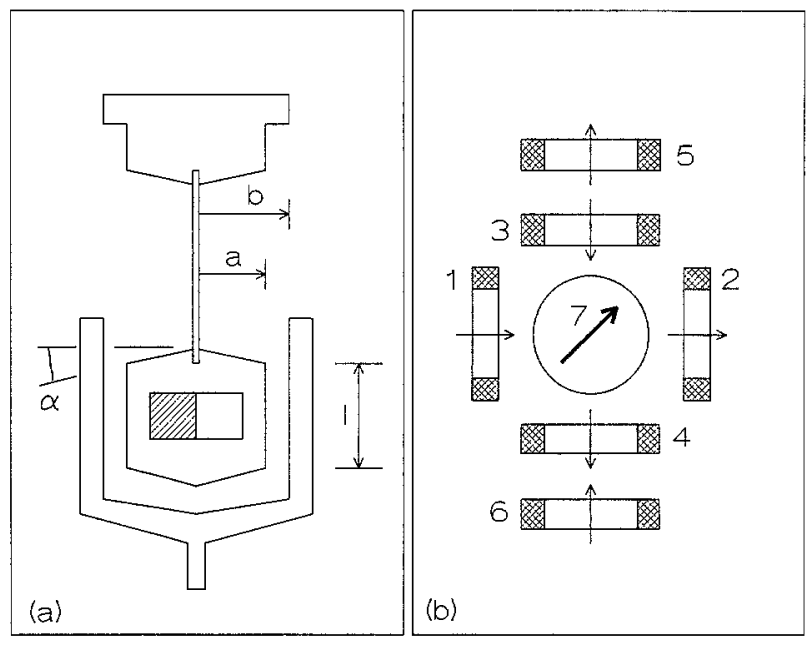

Fig. 2. Panel a: schematic view on the resonator set-up defining the radii $a$ and $b$, the length $l$, and the angle $\alpha$. Panel $b$ : the magnet-coil system: 1,2 excitation-, 3,4 detection-, 5,6 noise suppression coils, 7 the magnetic dipole direction; the small arrows indicate the winding sense of the coils

rotating sample holder; hence, the pendulum oscillates in a forced harmonic vibration, which is described by

$$
I \ddot{\vartheta}+\left(D+Z^{*}\right) \dot{\vartheta}+K \vartheta=M_{d r}+{ }^{0} M_{\mathrm{liq}},
$$

where $I$ is the moment of inertia of the lump, $K$ the torsional spring constant, $D$ the constant internal damping, $-Z * \dot{\vartheta}$ the dynamic part of the torque due to the surrounding liquid, $M_{\mathrm{dr}}$ the driving torque on the lump, and ${ }^{0} M_{\text {liq }}$ the steady-state part of the torque exerted by the liquid due to the viscometric part of the flow field. For the small angular amplitudes applied, the driving torque is proportional to the current through the excitation coils:

$$
M_{d r}=C_{1} e^{i \zeta} J_{\mathrm{exc}}=C_{1} e^{i \zeta} J_{0} e^{i \omega t} .
$$

Due to eddy currents induced in the lump itself this torque will not be completely in phase with the current $J_{\text {exc }}$, so a phase factor $e^{i \zeta}$ is introduced. The response of the lump is detected by two coils perpendicular to the excitation coils. The voltage induced by the vibrating lump in the detection coils $V_{\text {sig }}$ is for small angular displacements proportional to the angular velocity $\dot{\vartheta}$ :

$$
V_{\text {sig }}=C_{2} \dot{\theta}
$$

The excitation coils also directly induce a perturbing signal, a so-called cross talk: 


$$
V_{c r}=L \dot{J}_{\mathrm{exc}}
$$

where $L$ is the mutual inductance between excitation and detection coils; this inductance is not purely real but the imaginary part is much smaller then the real one. This cross talk is reduced as much as possible by the orthogonal coil setup. The complex transfer function $H^{*}=V_{\mathrm{det}} / J_{\mathrm{exc}}$, with $V_{\mathrm{det}}=V_{\mathrm{sig}}+V_{c r}$, is obtained by differentiation of Eq. (44) and substitution of Eqs. (45) - (47):

$$
H^{*}(\omega)=i \omega L+\frac{C_{1} C_{2} e^{i \zeta}}{R+D} \frac{1-i \chi(\omega)}{1+\chi^{2}(\omega)}
$$

where $\quad \chi(\omega)=\left(I \omega^{2}+X \omega-K\right) /[(R+D) \omega], R=$ $\operatorname{Re}\left(Z^{*}\right)$ and $X=\operatorname{Im}\left(Z^{*}\right)$. Due to the high quality factor $Q$ of the resonator $[X-\chi(R+D)]^{2} \ll 4 K I$ for low viscous fluids in the frequency range of interest, and the expression for $\chi(\omega)$ reduces to

$$
\chi(\omega) \simeq \frac{\omega-\omega_{0}}{\Delta \omega}
$$

where $\omega_{0}=(K / I)^{1 / 2}-X /(2 I)$ is the resonance frequency and $\Delta \omega=(R+D) /(2 I)$ is the bandwidth of the measured resonance curve [Eq. (53)], i.e., the half width half maximum of $1 /\left[1+\chi^{2}(\omega)\right]$. Measuring $\omega_{0}$ and $\Delta \omega$ both in liquid and in vacuum yields the information to obtain $Z^{*}=R+i X$ :

$$
\begin{aligned}
& R=2 I\left(\Delta \omega-\Delta \omega^{\mathrm{vac}}\right) \\
& X=2 I\left(\omega_{0}^{\mathrm{vac}}-\omega_{0}\right) .
\end{aligned}
$$

The results of the last two sections, Eqs. (43) and (50), establishes how $G_{\|}^{\prime}, G_{\|}^{\prime \prime}$ can be deduced from the measurable quantities $\omega_{0}-\omega_{0}^{\mathrm{vac}}$ and $\Delta \omega-\Delta \omega^{\mathrm{vac}}$.

\section{Description of the instrument and the measuring procedure}

The instrument discussed is an extension of the torsion pendula developed in our laboratory some years ago by Blom and Mellema [2]. At that time, much attention had been paid to the mechanical and thermal stability of the pendula and the excitation and detection system. Resonator details are copied for the present purpose, as is the magnet-coil system. The construction of a rotating sample holder is new. In addition, the detection is improved in order to suppress disturbing signals originating from rotating parts of the apparatus. Mechanical details are given in Fig. 3, while the electronic set-up is given in Fig. 4.

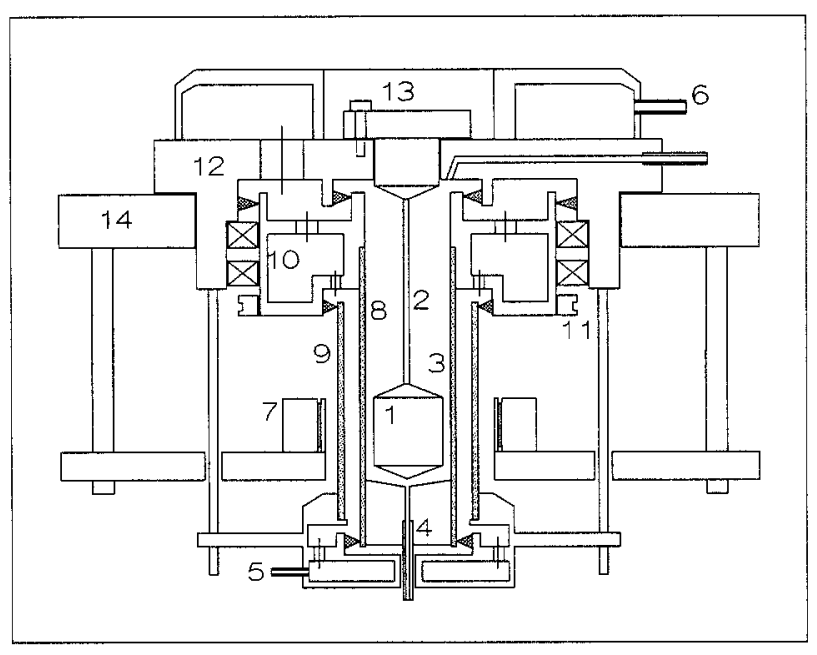

Fig. 3. Mechanical layout of the instrument: 1 resonator mass (lump), 2 torsional rod, 3 sample volume, 4 sample inlet, 5 thermostat water inlet, 6 thermostat water outlet, 7 coil, 8 rotating glass tube, 9 outer glass tube, 10 ball bearings, 11 gear-wheel for drive, 12 base plate, 13 resonator bracket, 14 frame

It is possible to easily exchange the resonator lumps by unscrewing the resonator bracket from the base plate at the upper side and removing the resonator along this side. Hence, the instrument can be operated at four different resonance frequencies. These frequencies are given in Table 1 together with the dimensions of the four resonators. The angle $\alpha$ is for all resonators $15^{\circ}$ and the inner radius of the sample

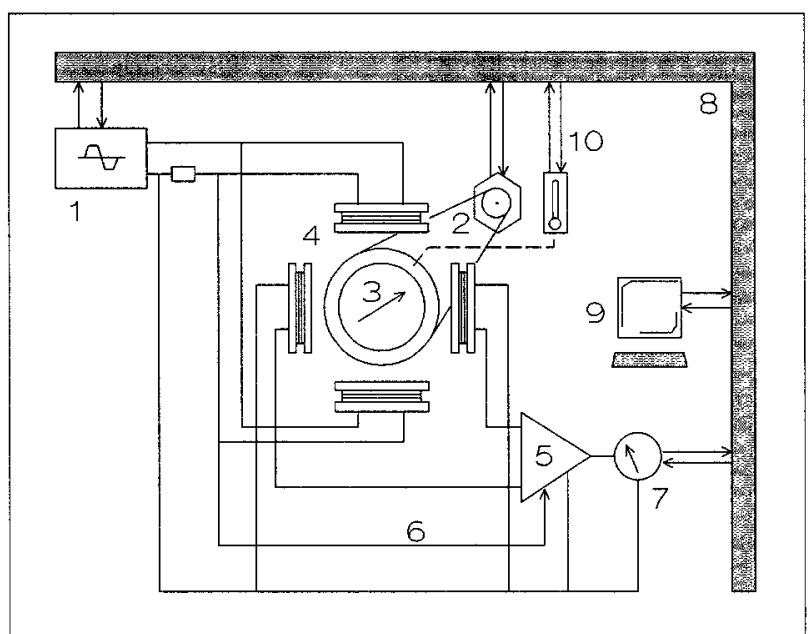

Fig. 4. The electronic set-up of the measuring system: 1 frequency synthesizer, 2 drive of the rotating sample holder, 3 lump with magnet, 4 excitation and detection coils, 5 lock-in amplifier, 6 reference signal, 7 in phase output, 8 IEEE-bus, 9 microcomputer, 10 thermostat 
Table 1. Pendulum dimensions and characteristics in vacuum at $25^{\circ} \mathrm{C}$

\begin{tabular}{|c|c|c|c|c|c|c|}
\hline $\begin{array}{l}\text { Pendulum } \\
\text { number }\end{array}$ & $\begin{array}{l}a \\
{[\mathrm{~mm}]}\end{array}$ & $\begin{array}{l}l \\
{[\mathrm{~mm}]}\end{array}$ & $\begin{array}{l}I \\
{\left[10^{-7} \mathrm{~kg} \mathrm{~m}^{2}\right]}\end{array}$ & $\begin{array}{l}\omega_{0} / 2 \pi \\
{[\mathrm{Hz}]}\end{array}$ & $\stackrel{Q}{=} \omega_{0} / 2 \Delta \omega$ & $\begin{array}{l}\frac{1}{\omega} \frac{\partial \omega}{\partial T} \\
{\left[10^{-5} \mathrm{~K}^{-1}\right]}\end{array}$ \\
\hline $\begin{array}{l}1 \\
2 \\
3 \\
4\end{array}$ & $\begin{array}{r}12.0 \\
12.0 \\
5.5 \\
5.5\end{array}$ & $\begin{array}{l}27.0 \\
27.0 \\
18.0 \\
18.0\end{array}$ & $\begin{array}{l}72 \\
72 \\
2.1 \\
2.1\end{array}$ & $\begin{array}{r}84.966 \\
283.211 \\
739.432 \\
2440.44\end{array}$ & $\begin{array}{r}7000 \\
15000 \\
9000 \\
15000\end{array}$ & $\begin{array}{l}-1.4 \\
-1.4 \\
-1.7 \\
-3.0\end{array}$ \\
\hline
\end{tabular}

holder $b$ is $14 \mathrm{~mm}$. The amount of sample needed is $15 \cdot 10^{-6} \mathrm{~m}^{3}$ for the two lower frequencies and $25 \cdot 10^{-6} \mathrm{~m}^{3}$ for the other frequencies.

In Sect. 2 surface loading $k^{\prime \prime} h \gg 1$ was assumed. From Eq. (20), one obtains in the case of nearly surface loading:

$$
Z_{0}^{*} \simeq\left(\frac{\varrho \omega}{k^{*}}\right)\left(1+2 e^{-2 i k^{*} h}\right)
$$

With the last equation, one is able to estimate the range of $\eta^{\prime}$ and $\eta^{\prime \prime}$ for which the error introduced by assuming pure surface loading is smaller than the experimental error. In the next section the experimental error will be considered. The relative experimental error in $\eta^{\prime}$ and $\eta^{\prime \prime}$ appears to be about $1 \%$ of $\left|\eta^{*}\right|$ or more for the four pendula, so the relative error due to the approximation must be smaller than $1 \%$ :

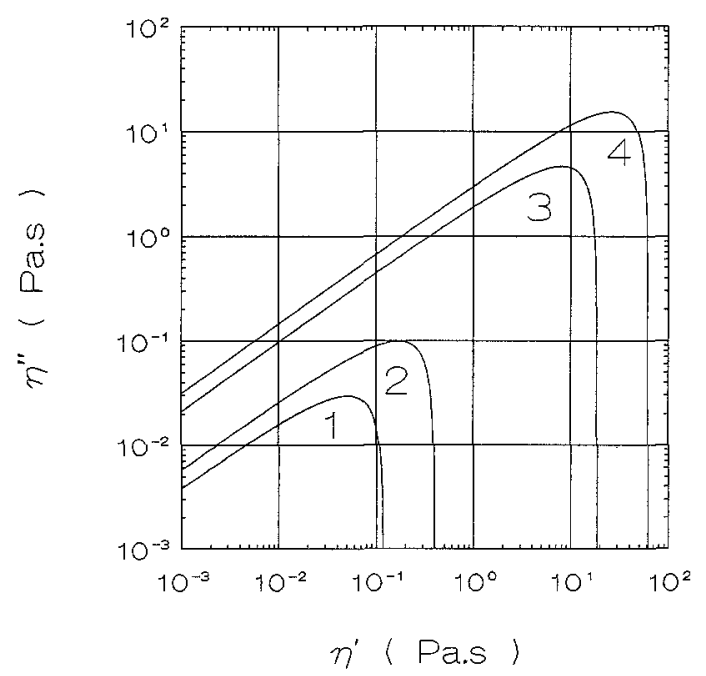

Fig. 5. The operational range of the pendula in the $\eta^{\prime}-\eta^{\prime \prime}$ plane. For the area under the plotted curves the error due to the assumption of surface loading is smaller than $1 \%$. The numbers indicate the pendulum number
$\left|\Delta \eta^{*}\right| /\left|\eta^{*}\right|<0.01$. From Eqs. (16) and (21) and with $i \omega \eta^{*}=G^{*}$, one obtains: $i \omega \varrho \eta^{*}=\left(Z_{0}^{*}\right)^{2}$, so $\left|\Delta \eta^{*} / \eta^{*}\right|=2\left|\Delta Z_{0}^{*} / Z_{0}^{*}\right|$, and with Eq. (51): $\left|\Delta \eta^{*} / \eta^{*}\right|=4 \exp (-2 h / \delta)<0.01, \quad$ with $\delta=$ $\left[\omega \varrho\left(\left|\eta^{*}\right|-\eta^{\prime \prime}\right) /\left(2\left|\eta^{*}\right|^{2}\right)\right]^{-1 / 2}$ the penetration depth, which means that $h / \delta$ has to be $\geq 3$. In Fig. 5 the permissible ranges for $\eta^{\prime}$ and $\eta^{\prime \prime}$ are indicated for the four different pendula by the region below the plotted curves, assuming a fluid density $\varrho=1000 \mathrm{~kg} / \mathrm{m}^{3}$.

The double-walled sample holder is constructed from glass tubes. Between these tubes water circulates for temperature control. The inner tube is able to rotate and is driven by a tacho-controlled motor, mounted $1 \mathrm{~m}$ below the resonator in order to prevent electromagnetic disturbances. The motor is coupled to the inner tube of the sample holder by a long vertical shaft, a belt and a gear-wheel made of polyoxymethylene. The rotational transmission ratio from motor to sample holder is 20:72. The motor can be controlled between 0.07 and $4 \mathrm{rpm}$, resulting in an angular velocity of the sample holder between 0.1 and $7 \mathrm{rad} \mathrm{s}^{-1}$. Assuming a power-law dependence of the viscometric shear stress $\tau_{0}$ on the steady shear rate, $\dot{\gamma}_{0}: \tau_{0}=c \dot{\gamma}_{0}^{n}$, the relation between the angular velocity $\Omega$ and the steady shear rate on the side of the lump is given by:

$$
\dot{\gamma}_{0}=\frac{2 b^{2 / n} \Omega / n}{b^{2 / n}-a^{2 / n}},
$$

thus, for a Newtonian liquid $(n=1) \dot{\gamma}_{0}$ is adjustable from 0.75 to $50 \mathrm{~s}^{-1}$ for resonators 1 and 2 , and from 0.24 to $16 \mathrm{~s}^{-1}$ for resonators 3 and 4 .

As mentioned, the magnet-coil system is copied from the older pendula, the two additional detection coils for far field noise reduction included. Only the distances to the lump are slightly different, so one has to adjust the values for $C_{2}$ as measured by Blom and Mellema [2]: $C_{2}=6.6 \cdot 10^{-3} \mathrm{Vs} / \mathrm{rad}^{2}$ for resonators 1 and $2, C_{2}=1.6 \cdot 10^{-3} \mathrm{Vs} / \mathrm{rad}^{2}$ for resonators 3 and 4 . These corrected values are, within $5 \%$, equal to values 
obtained from a calculation of the magnetic flux through the detection coils. With a lowest detectable $(10 \%$ accuracy) output voltage of $\simeq 10 \mu \mathrm{V}$, this means that the smallest angular amplitude is $\vartheta_{0} \simeq 3 \cdot 10^{-6} \mathrm{rad}$ at $85 \mathrm{~Hz}$ and $\vartheta_{0} \simeq 1.5 \cdot 10^{-6}$ at $740 \mathrm{~Hz}$, thus, the smallest operational shear amplitude $\hat{\gamma}$ becomes $3 \cdot 10^{5}$ and $1.5 \cdot 10^{-5}$, respectively.

The current through the excitation coils is generated by the frequency synthesizer (HP 3325 A). The settings of this synthesizer are controlled by a microcomputer (Olivetti M240). To eliminate the influence of the cross talk as much as possible, the output voltage is measured using a lock-in amplifier (PAR 5204). As reference signal the current $J_{\text {exc }}$ is used. During a frequency scan the amplitude $J_{0}$ of this current is constant within $0.5 \%$. By phase adjustment of the lock-in, its output $U(\omega)$ can be made symmetrical around the resonance frequency $\omega_{0}$. In that case the adjusted phase is equal to $-\zeta$ [see Eq. (48)] and:

$$
\begin{aligned}
U(\omega) & =\operatorname{Re}\left(H^{*}(\omega) e^{-i \zeta}\right) \\
& =\frac{C_{1} C_{2}}{R+D} \frac{1}{1+\chi^{2}(\omega)}+\omega L \sin \zeta .
\end{aligned}
$$

The small term $\omega L \sin \zeta$ is constant within $1 \%$ in the relatively small frequency range considered. The output signal of this lock-in amplifier is read by the microcomputer, which also controls the motor of the rotating sample holder.

In a typical measurement resonance curves are taken for a certain number of steady shear rates. The

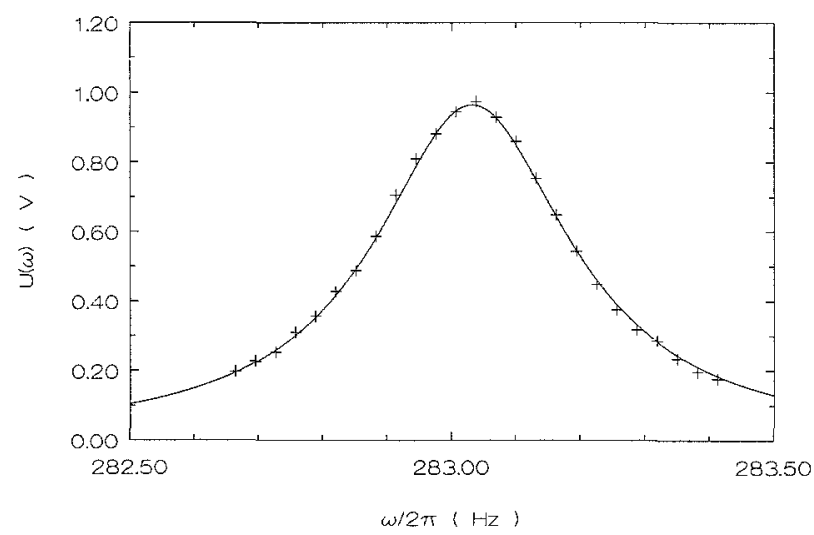

Fig. 6. A typical resonance curve as measured with pendulum 2 at $\dot{\gamma}_{0}=27.9 \mathrm{~s}^{-1}$ for 1-butanol. The + symbols indicate the measured output signal from the lock-in, while the curve represents the best least squares fit to the data points which resulted in $\omega_{0} / 2 \pi=283.031 \pm 0.001 \mathrm{~Hz}$ and $\Delta \omega / 2 \pi=0.185 \pm 0.003 \mathrm{~Hz}$, respectively reference measurements were performed at $\dot{\gamma}_{0}=0$ in air instead of in vacuum, hence, to obtain the bandwidth in vacuum a small correction is needed on the order of one-fifth of the bandwidth measured in air. A typical resonance curve is shown in Fig. 6, where the lock-in output signal $U(\omega)$ has been measured for $\omega$ between $\omega_{0}-2 \Delta \omega$ and $\omega_{0}+2 \Delta \omega$. The theoretical curve [Eq. (53)] is fitted to the experimental data points, using a least square fit procedure based on the Levenberg-Marquardt method, as described by Press et al. [10]. From such a fit values for $\omega_{0}$ and $\Delta \omega$ are obtained. With those values and the values for $\omega_{0}^{\text {vac }}$ and $\Delta \omega^{\mathrm{vac}}$, as obtained from the reference measurement, $G_{\|}^{\prime}\left(\dot{\gamma}_{0}, \omega\right)$ and $G_{\|}^{\prime \prime}\left(\dot{\gamma}_{0}, \omega\right)$ are calculated or, equivalently, $\eta_{\|}^{\prime}\left(\dot{\gamma}_{0}, \omega\right)$ and $\eta_{\|}^{\prime \prime}\left(\dot{\gamma}_{0}, \omega\right)$. Typical measuring time of one frequency curve is about $5 \mathrm{~min}$ for the lowest frequency and less than $2 \mathrm{~min}$ for the other frequencies.

\section{Calibration and performance}

The validity of Eqs. (43) and (50) has been checked experimentally for the four pendula. In Table 1 the characteristics of the pendula in vacuum are given. Also listed is the thermal stability. This stability is of great importance for the accuracy of the instrument. If one wishes to determine, for instance, the viscosity of water within $1 \%$, a variation of the frequency $\Delta \omega / \omega$ as small as $2 \cdot 10^{-6}$ is needed. Thus, due to the high thermal stability a variation of $0.1{ }^{\circ} \mathrm{C}$ in the temperature of the instrument is allowed.

Table 2. Properties of the Newtonian calibration liquids at $25^{\circ} \mathrm{C}$

\begin{tabular}{lcc}
\hline Sample & $\begin{array}{l}\text { Density } \\
{\left[\mathrm{kg} \cdot \mathrm{m}^{-3}\right]}\end{array}$ & $\begin{array}{c}\text { Viscosity } \\
{[\mathrm{mPa} \cdot \mathrm{s}]}\end{array}$ \\
\hline hexane & 653 & 0.294 \\
ethanol & 790 & 1.1 \\
1-butanol & 807 & 2.6 \\
dibutylphthalate (DBP) & 1040 & 16.2 \\
di(ethyl-hexyl)phthalate (DEHP) & 978 & 58.0 \\
\hline
\end{tabular}

Table 3. The calculated and experimentally obtained values for the geometrical constants

\begin{tabular}{lllll}
\hline $\begin{array}{l}\text { Pendulum } \\
\text { number }\end{array}$ & $\begin{array}{l}A_{\text {calc }} \\
{\left[\mathrm{kg} \cdot \mathrm{m}^{-2}\right]}\end{array}$ & $\begin{array}{l}A_{\text {meas }} \\
{\left[\mathrm{kg} \cdot \mathrm{m}^{-2}\right]}\end{array}$ & $\begin{array}{l}E_{\text {calc }} \\
{\left[\mathrm{m}^{-1}\right]}\end{array}$ & $\begin{array}{l}E_{\text {meas }} \\
{\left[\mathrm{m}^{-1}\right]}\end{array}$ \\
\hline 1 & $40 \pm 1$ & $39.50 \pm 0.03$ & $102 \pm 2$ & $110 \pm 4$ \\
2 & $40 \pm 1$ & $39.51 \pm 0.04$ & $102 \pm 2$ & $99 \pm 5$ \\
3 & $19.3 \pm 0.5$ & $19.29 \pm 0.02$ & $235 \pm 5$ & $257 \pm 8$ \\
4 & $19.3 \pm 0.5$ & $19.34 \pm 0.03$ & $235 \pm 5$ & $210 \pm 20$ \\
\hline
\end{tabular}


With the help of five different Newtonian liquids the geometrical constants $A$ and $E$ [see Eq. (43)] are determined. From Eqs. (41) and (42), one obtains for a Newtonian liquid:

$$
A\left(\Delta \omega-\Delta \omega^{\mathrm{vac}}\right)=R_{0}+E \eta
$$

where $R_{0}=X_{0}(\varrho \omega \eta / 2)^{1 / 2}$. At $25.0^{\circ} \mathrm{C}$ and zero steady shear flow, the resonance frequencies and bandwidths of the resonators immersed in the calibration liquids are determined. From the measured bandwidths the constants $A$ and $E$ are calculated with a least square fit of Eq. (54). The results are given in Table 3, together with the calculated values for $A$ and $E$. For this calculation the data of Table 1 have been used. The agreement between calculated and measured values is quite good for all the pendula. The rather large error in the measured $E$-values does not influence the accuracy of $G$ * very much, because the value of the correction term $i E A Z * / \omega \varrho I$ in Eq. (43) is, at most, 0.05 .

Next, the reproducibility of the resonators was studied with the help of the Newtonian liquids, both for $\dot{\gamma}_{0}=0$ and for $\dot{\gamma}_{0} \neq 0$, by repeatedly measuring at fixed $\dot{\gamma}_{0}$ values. The experimental accuracy has been estimated from the measured reproducibility. In the case of $\dot{\gamma}_{0} \neq 0$ the whole available range of the shear rate was scanned. These results are collected in
Table 4. Inspection of this table shows that, for $\dot{\gamma}_{0}=0$, an accuracy of better than $3 \%$ in the viscosity can be obtained, while for $\dot{\gamma}_{0} \neq 0$ the accuracy is better than $5 \%$. From the experimental data also a value for $\eta^{\prime \prime}$ can be calculated, which, of course, has to be zero for Newtonian liquids. From the spread in the obtained values for $\eta^{\prime \prime}$ an accuracy of about $3 \%$ in case of $\dot{\gamma}_{0}=0$ and about $5 \%$ in case of $\dot{\gamma}_{0} \neq 0$ is obtained for $\eta^{\prime \prime}$ relative to $\left|\eta^{*}\right|$. During the measurements neither the values of the viscosities nor their accuracies showed any correlation with the value of the steady shear rate. Analyzing the dependence of the accuracy of the viscosity upon the uncertainty in $R$ and $X$, one is able to estimate by means of propagation of errors the accuracy in $\eta^{\prime}$ and $\eta^{\prime \prime}$ for a viscoelastic liquid: both $\Delta \eta^{\prime}$ and $\Delta \eta^{\prime \prime}$ are smaller than $3 \%$ of $\left|\eta^{*}\right|$ without steady shear flow and smaller than $5 \%$ with a steady shear flow.

\section{Discussion and conclusion}

With the analysis given in Sect. 2, we are able to handle the problem of shear wave propagation in $a$, due to the steady-state shear flow, non uniform medium. Hence, also in a steady-state flow, the complex viscosity of a liquid can be measured using the impedance concept.

Table 4. The reproducibility of the values for $\eta$ of the calibration liquids with and without steady shear flow at $25^{\circ} \mathrm{C}$

\begin{tabular}{llcc}
\hline Sample & $\begin{array}{l}\text { Pendulum } \\
\text { number }\end{array}$ & $\begin{array}{l}\text { Viscosity }\left(\dot{\gamma}_{0}=0\right) \\
{[\mathrm{mPa} \cdot \mathrm{s}]}\end{array}$ & $\begin{array}{c}\text { Viscosity }\left(\dot{\gamma}_{0} \neq 0\right) \\
{[\mathrm{mPa} \cdot \mathrm{s}]}\end{array}$ \\
\hline hexane & 1 & $0.30 \pm 0.01$ & $0.31 \pm 0.01$ \\
& 2 & $0.30 \pm 0.01$ & $0.31 \pm 0.01$ \\
& 3 & $0.32 \pm 0.01$ & $0.32 \pm 0.01$ \\
ethanol & 4 & $0.30 \pm 0.01$ & $0.30 \pm 0.02$ \\
& 1 & $1.14 \pm 0.08$ & $1.1 \pm 0.1$ \\
& 2 & $1.12 \pm 0.08$ & $1.1 \pm 0.1$ \\
1-butanol & 3 & $1.15 \pm 0.08$ & $1.2 \pm 0.1$ \\
& 4 & $1.15 \pm 0.05$ & $1.1 \pm 0.1$ \\
& 1 & $2.65 \pm 0.07$ & $2.6 \pm 0.1$ \\
DBP & 2 & $2.62 \pm 0.08$ & $2.62 \pm 0.04$ \\
& 3 & $2.70 \pm 0.04$ & $2.6 \pm 0.2$ \\
& 4 & $2.60 \pm 0.04$ & $2.6 \pm 0.2$ \\
DEHP & 1 & $16.2 \pm 0.2$ & $16.2 \pm 0.3$ \\
& 2 & $16.3 \pm 0.1$ & $16.3 \pm 0.2$ \\
& 3 & $16.3 \pm 0.2$ & $16.3 \pm 0.3$ \\
& 4 & $16.2 \pm 0.2$ & $5.2 \pm 0.4$ \\
& 1 & $58.1 \pm 0.7$ & $58 \pm 1$ \\
& 2 & $58.3 \pm 0.5$ & $58 \pm 2$ \\
& 3 & 58 & $58 \pm 3$ \\
\hline
\end{tabular}


In the previous section it has been demonstrated that the apparatus operates well for low viscous fluids, as indicated in Fig. 5. The accuracy in both $\eta^{\prime}$ and $\eta^{\prime \prime}$ is about $3 \%$ of $\left|\eta^{*}\right|$ at equilibrium, and about $5 \%$ of $\left|\eta^{*}\right|$ in case of a steady shear flow, so the smallest elastic effect detectable $G^{\prime}$ is also about $3 \%$, respectively, $5 \%$ of $\omega\left|\eta^{*}\right|$.

By application of a lock-in technique the detection system is considerably improved and angular amplitudes as small as $2 \cdot 10^{-6} \mathrm{rad}$ are detectable, even with a rotating sample holder. Hence, also materials with a small linear regime (as expected for weakly flocculating dispersions for instance) can be handled properly. The variability of the oscillatory shear amplitude with a factor of about 50 enables to check this linearity. Next, we will extent the range of the instrument towards higher and lower shear rates.

\section{Acknowledgements}

The authors wish to thank Mr. E. Altena and Mr. G. Beukema for their technical support, and Mr. F. Ganzevles for his contribution to the improvement of the detection system and the calibration of the apparatus. This work is part of the research program of the Foundation for Fundamental Research on Matter (FOM) with financial support from the Netherlands Organization for Pure Research (NWO).

\section{References}

1. Abramowitz M, Stegun IA (1970) Handbook of mathematical functions. Dover Publications, New York

2. Blom C, Mellema J (1984) Torsion pendula with electromagnetic drive and detection system for measuring the complex shear modulus of liquids in the frequency range $80-2500 \mathrm{~Hz}$. Rheol Acta 23:98-105

3. Booij HC (1966) Influence of superimposed steady shear flow on the dynamic properties of non-Newtonian fluids. Rheol Acta 5:215-221, 222-227
4. Booij HC (1968) Influence of superimposed steady shear flow on the dynamic properties of non-Newtonian fluids. Rheol Acta 7:202-209

5. Darby R (1976) Viscoelastic Fluids. Marcel Dekker Inc, New York

6. Elmore WC, Heald MA (1969) Physics of waves. McGraw-Hill Book Company, New York

7. Ferry JD (1980) Viscoelastic properties of polymers. John Wiley \& Sons, New York

8. Papenhuijzen JMP (1971) Superimposed steady and oscillatory shear in dispersed systems. Rheol Acta $10: 493-502,503-507$

9. Pipkin AC, Owen DR (1967) Nearly viscometric flows. Phys Fluids 10:836-843

10. Press WH, Flannery BP, Teukolsky SA, Vetterling WT (1986) Numerical recipes, the art of scientific computing. Cambridge University Press, Cambridge

11. Schowalter WR (1978) Mechanics of non Newtonian Fluids. Pergamon Press, Oxford

12. Schrag JL, Johnson RM (1971) Application of the Birnboim multiple lumped resonator principle to viscoelastic measurements of dilute macro molecular solutions. Rev Sci Instr 42:224-232

13. Smeulders JBAF, Blom C, Mellema J (1990) Linear viscoelastic study of lipid vesicle dispersions: hard sphere behaviour and bilayer surface dynamics. Phys Rev A 15 42:3483-3498

14. Truesdell C, Noll W (1965) Non linear field theories in mechanics, in Springer Encyclopedia of Physics III/3. Springer-Verlag, Berlin

15. Volterra V (1959) Theory of functionals and of integral and integro-differential equations. Dover Publications, New York

16. Van der Werff JC, de Kruif CG, Blom C, Mellema J (1989) Linear viscoelastic behaviour of dense hardsphere dispersions. Phys Rev A 39:795-807

(Received July 16, 1991;

in revised form December 19, 1991)

\section{Correspondence to:}

Dr. D. van den Ende

Rheology Group, Faculty of Applied Physics

University of Twente, P.O. Box 217

7500 AE Enschede, The Netherlands 